\title{
Image-Guided Focused Ultrasound for the Treatment of Bone Metastases: Current Status and Future Direction
}

\author{
Mark D. Hurwitz • Nicholas G. Zaorsky
}

Published online: 5 May 2013

(c) Springer Science+Business Media New York 2013

\begin{abstract}
Bone metastases are a pervasive problem in oncology. For many of these patients, pain due to osseous metastases is the predominant factor negatively influencing quality of life. High-intensity focused ultrasound (FUS) therapy is a novel technology used for the treatment of bone metastases. FUS can be done with either ultrasound or magnetic resonance guidance (USgFUS or MRgFUS respectively). We review the outcomes and toxicity of current studies using USgFUS and MRgFUS for the treatment of bone metastases. A recently completed phase III trial has confirmed statistically and clinically significant benefit in pain control and quality of life for patients with painful bone metastases who were not candidates for radiation therapy. Future areas of research include expansion of sites suitable for MRgFUS treatment, applicability of MRgFUS as a primary treatment for painful bone metastases either as standalone therapy or in combination with radiation, and development of conformal treatment devices.
\end{abstract}

Keywords High-intensity focused ultrasound ablation . Magnetic resonance imaging - Neoplasm metastasis . Radiology · Ultrasonography

\section{Introduction}

Bone metastases are a pervasive and daunting problem in oncology. Bone is the third most common organ involved

M. D. Hurwitz $(\bowtie) \cdot$ N. G. Zaorsky

Department of Radiation Oncology, Bodine Center for Cancer

Treatment, Jefferson Medical College of Thomas Jefferson

University, 111 South 11th Street, Philadelphia, PA 19107, USA

e-mail: mark.hurwitz@jeffersonhospital.org

N. G. Zaorsky

e-mail: nicholaszaorsky@gmail.com by metastatic disease after lung and liver [1]. Autopsy studies have revealed that amongst the most prevalent cancers most patients with advanced disease develop bone metastases. In fact, as many as $90 \%$ of breast and prostate cancer patients who die from their disease have evidence of bone metastases at the time of death [1-4]. As systemic therapies have become increasingly effective, a concomitant increase in the prevalence of bone metastases has also occurred. For many of these patients, pain due to osseous metastases is the predominant factor negatively influencing quality of life. In the case of prostate cancer and breast cancer in particular, these patients often suffer for years with pain and disability due to bone metastases.

The need for effective new therapies for metastatic bone pain is clear. Studies indicate that approximately half of patients with bone metastases receive only temporary pain relief with treatment $[5,6]$ and this relief is seldom complete. Radiation therapy is considered the standard intervention for focal pain from osseous metastases when risk of fracture is not imminent. Prior series, however, indicate up to approximately a third of patients treated with radiation do not experience pain relief [1, 7]. Notably, a Radiation Therapy Oncology Group Phase III Trial, RTOG 97-14, revealed $34 \%$ of patients with bone metastases do not obtain significant pain relief with radiation and only $17 \%$ achieve complete response [1]. Retreatment rates are generally reported in the range of $8-20 \%$ [8]; however, pain often recurs in patients who are able to undergo retreatment leaving them with limited options.

Other therapies for bone pain generally yield modest results and can be associated with significant side effects. Many patients with bone pain are chronically managed with narcotic medications which are associated with widely recognized side effects including malaise, mental status changes, nausea, and constipation. Chemotherapy and hormonal 
interventions often have limited benefit in managing active bone pain and are frequently associated with significant side effects. Surgery can be useful for prophylactic treatment of extensive disease in weight bearing bones or stabilization after fracture provided patients are suitable surgical candidates including sufficient life expectancy. Radiation therapy is typically used following surgery.

\section{Pain Assessment: Challenges and Methods}

Pain is a subjective experience and therefore establishing benefit of interventions for alleviation of pain has proven problematic. The standard definition for pain, as developed by the International Association for the Study of Pain is: "An unpleasant sensory and emotional experience normally associated with tissue damage or described in terms of such damage." The impact of pain is typically global. Quality of life can be impacted physically, emotionally, socially and financially. In addition, definition of clinically meaningful pain relief and impact on quality of life apart from statistically significant changes has also proven challenging given the dependence on patient reported outcomes. To assess pain and impact on quality life several instruments have been developed, validated, and endorsed by consensus panels and working groups. A consensus meeting of the Initiative on Methods, Measurement, and Pain Assessment in Clinical Trials (IMMPACT) resulted in definition of clinically significant changes in widely used tools to assess pain and its impact including the numeric rating scale (NRS) and brief pain inventory-quality of life (BPI-QOL). Descriptions of commonly used instruments are described below:

- Numerical rating scales (NRS): Developed by the World Health Organization (WHO) in 1994 [9], the NRS is a 11-point pain intensity scale $(0=$ no pain and $10=$ worst possible pain) which is widely used for the assessment of cancer pain and have been used to investigate the effectiveness of radiation therapy to relieve pain resulting from bone metastases $[10,11]$. The NRS worst pain score is highly correlated with quality of life and with mild, moderate and severe pain categories [10-12]. A 2-point change on the NRS of pain intensity is accepted as a clinically important change in pain in patients with bone metastases qualitatively correlating with patients expressing that their pain is "much improved" with a 4-point decline equating with "very much improved". (See description of overall treatment effect scale [OTE] below)

- Visual analogue score (VAS): The visual analog scale for pain (VAS) is a type of rating scale $(0=$ death; $100=$ perfect or optimal health), in which the patient marks a point on the line that matches the amount of pain he or she feels [13]. Changes in this score are widely used to estimate the efficacy of various treatments/medications for pain palliation. The VAS is conceptually similar to the NRS.

- The overall treatment effect scale (OTE) is an anchorbased method used to examine the relationship between the NRS score and an independent measure (or anchor) to explain the meaning of a particular change in a context that can be meaningful to clinicians. The OTE can be used to capture patient estimates of their global rating of change in pain at the treatment site to anchor change scores on a given instrument such as the NRS [11]. Subjects are asked to rate their perception of change, using a global rating scale to indicate whether they have improved, stayed the same, or worsened.

- Brief pain inventory-quality of life (BPI-QOL): The BPI-QOL is a questionnaire that enquires how much pain interferes with subjects' function in seven different areas of daily life including activity, mood, walking ability, normal work, relations with other people, sleep, and enjoyment of life. A 0-10 numerical rating scale, whereby 0 represents "Does not interfere" and 10 represents "Completely interferes." Factor analyses of responses show that the 7 interference items load together onto a single factor and that a one point change is considered clinically significant $[5,6,14]$.

\section{Clinical Experience with Focused Ultrasound}

Image Guidance for Therapy

High-intensity focused ultrasound (FUS) therapy can be done with either ultrasound or magnetic resonance guidance (USgFUS or MRgFUS, respectively). Published outcomes and toxicities of these techniques are displayed in Table 1. The principal advantages of US guidance are low cost and accessibility as MR is not required. MR (Fig. 1), however, has several advantages over US in regard to both imaging and treatment monitoring. MR allows for complete visualization of the therapeutic ultrasound beam path and more detailed imaging of the target. Temperature can also be measured with MR using proton resonance frequency shift (PRF; Fig. 2). Temperature measurement provides a quantification of thermal dose, which is useful both for ensuring adequate temperatures are achieved at the target while normal structures are kept at a safe temperature throughout the procedure.

While PRF cannot be used to measure temperature in bone, measurement of temperature at the bone/soft tissue interface has proven adequate in the actual therapeutic 


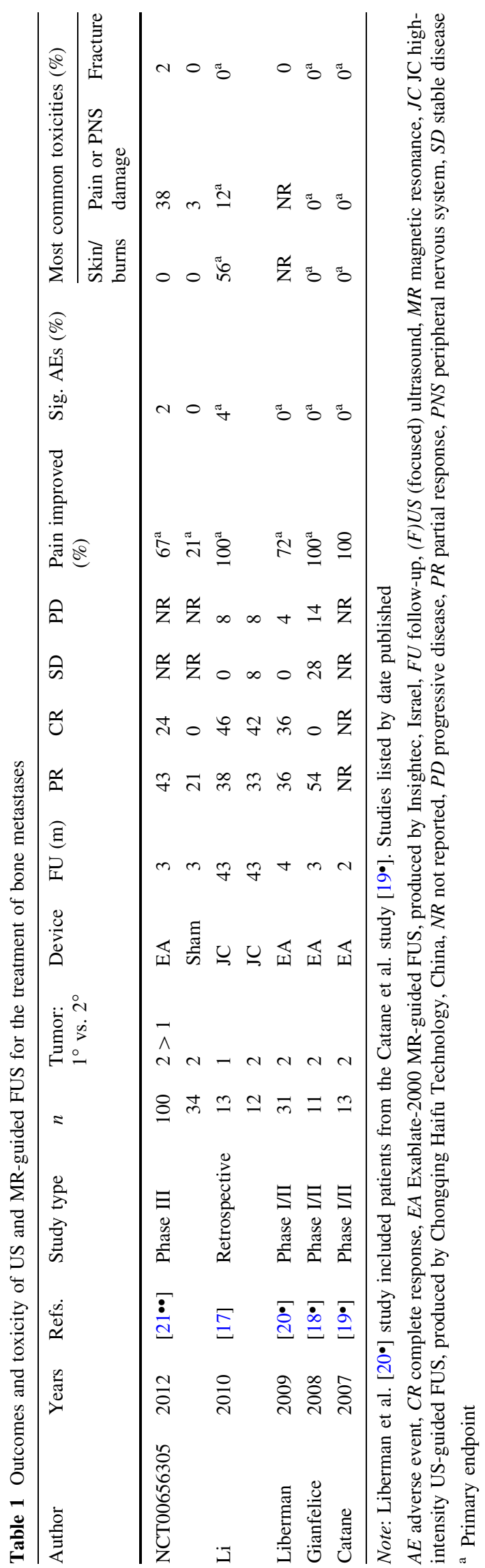

setting. An additional advantage of MR guidance is that the extent of treatment can be assessed before the patient is removed from the MR machine to ensure that treatment goals have been met by assessing characteristic changes in ablated bone with magnetic resonance imaging.

\section{Ultrasound Guided Focused Ultrasound (USgFUS) for Bone Metastases}

Three studies (two prospective $[15,16]$ and one retrospective [17]) using USgFUS for the treatment of primary bone tumors have been published. Among them, 100 patients were treated with USgFUS (using the Model JC from Chongqing Haifu Technology, China) with or without chemotherapy as primary or salvage therapy. The follow-up times ranged from 37 to 68 months (median, 43 months). The primary outcome measures were tumor response [15] and incidence of adverse events [16, 17]. After a median follow up time of 43 months, the average incidence of severe adverse events (i.e. RTOG Grades 3-4) was $12 \%$; Grade 2, $20 \%$; and Grade 1, $42 \%$. The most common toxicities included skin burns (average incidence, $45 \%$ ), followed by neuropathic pain (18\%), and bone fracture $(2.7 \%)$. Ligamentous injury, epiphysiolysis, and infection are rare and have occurred in less than $2 \%$ of patients in one prospective study [15]. Pain secondary to tumor improved in $100 \%$ of patients, though pain grading systems varied. The average 5-year rate of overall survival of this heterogeneous group of patients was 52 months. All studies used RECIST criteria to grade tumor response; the average rate of partial response was $39 \%$; complete response, $50 \%$; progressive disease, $7.3 \%$. These results, while difficult to extrapolate to metastatic disease, are sufficiently intriguing to support the enrollment of patients onto well designed trials for the use of USgFUS for secondary bone tumors.

\section{Magnetic Resonance Guided Focused Ultrasound (MRgFUS) for Bone Metastases}

Initial trials of MRgFUS for treatment of painful bone metastases yielded promising results. Two phase I/II trials $[18 \bullet, 19 \bullet]$ for treatment of osseous metastases revealed an excellent safety profile and high rates of pain response even as second line therapy. The results of these two trials coupled with additional patients treated under the same treatment protocol were summarized in a subsequent report. Thirty-six procedures were performed on 31 subjects [20•]. Twenty-one had previously received radiation to the treated site. Twenty-five subjects were available for 3 month post-treatment follow-up. Seventy-two percent of patients reported significant pain improvement. Average VAS pain scores were reduced from 5.9 prior to treatment 
Fig. 1 Schematic of treatment set up for MRgFUS bone metastases treatment with table mounted applicator. Courtesy Insightec, Inc

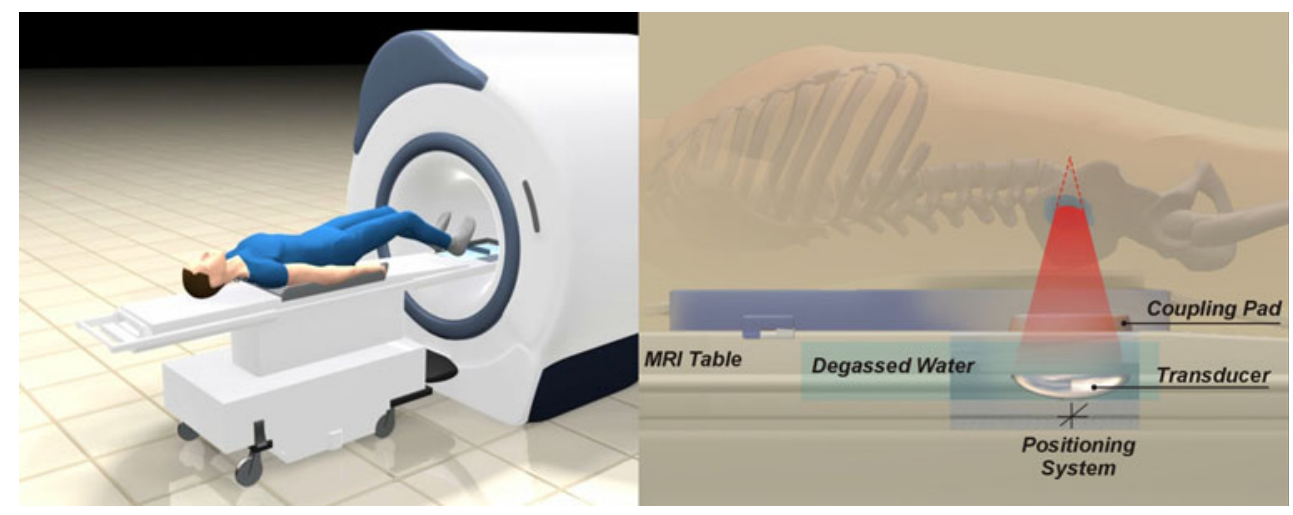

A

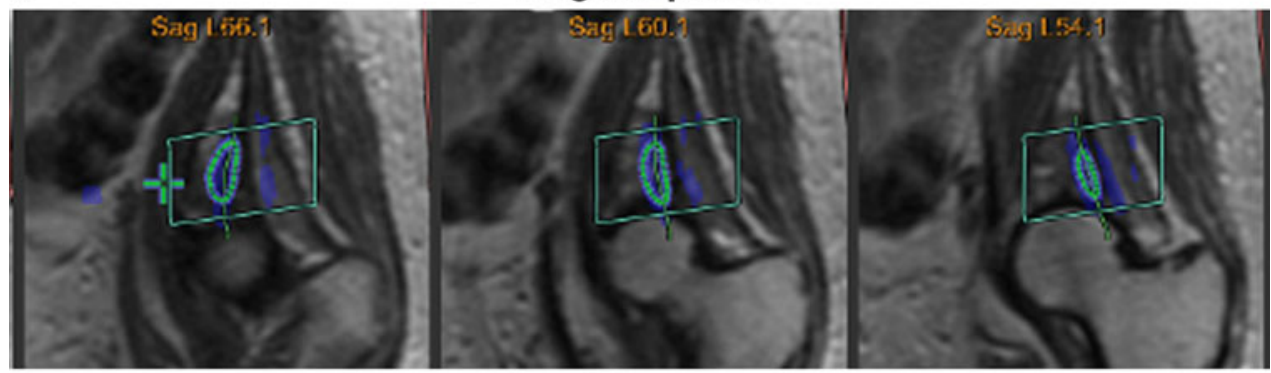

axial plane

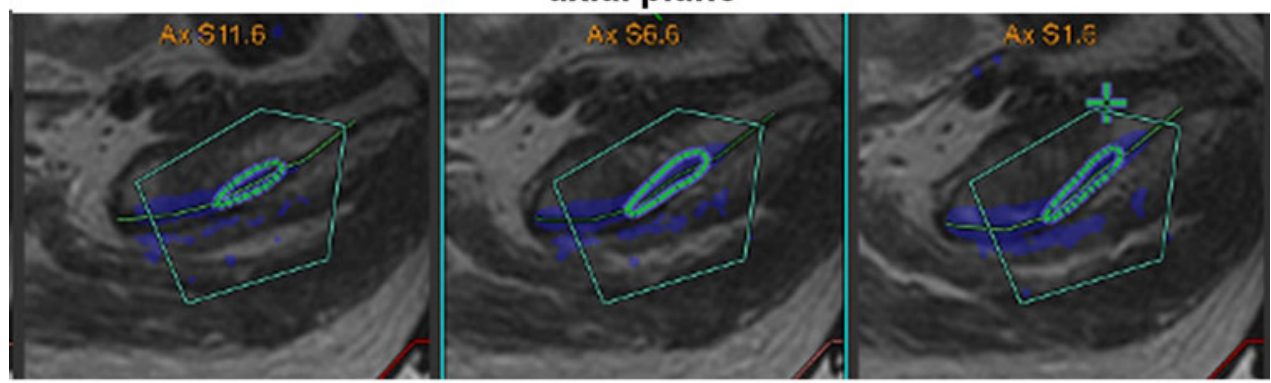

B T $\left({ }^{\circ} \mathrm{C}\right)$

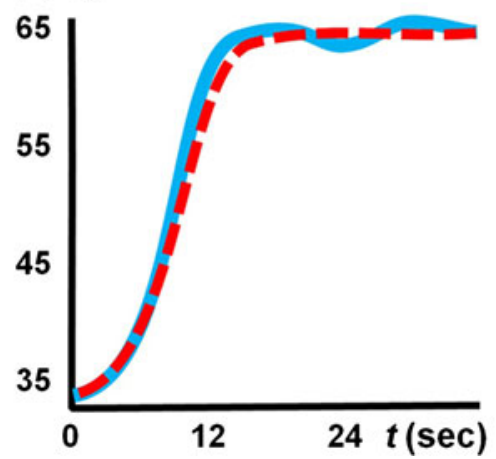

to 1.8 three months post treatment. No device-related serious adverse events occurred.

A phase III trial has now been completed [21••]. The primary objective was to evaluate effectiveness of MRgFUS thermal ablation treatment as compared to sham treatment in which no energy was delivered, to alleviate pain caused by osseous metastases or multiple myeloma in subjects who were not appropriate candidates for radiation therapy. Additional objectives included assessment of impact on quality of life and toxicity. 
Eligible subjects had distinguishable pain at one tumor site, and worst pain NRS score of $\geq 4$ despite optimization of medication and other measures. Subjects with $\leq 5$ painful bone lesions were eligible provided the targeted lesion had an NRS score $\geq 2$ points greater than other lesions. The target lesion had to be located in ribs, extremities excluding joints, pelvis, shoulders, or posterior aspects of spinal vertebra below L2 and device accessible. Mirel's fracture risk score was $\leq 7$. Subjects were randomized 3:1 to MRgFUS or sham treatment. Subjects remained blinded through month 3 , except nonresponding sham-treated subjects were allowed cross-over MRgFUS treatment after 2 weeks.

The results of an interim analysis have now been reported [21••]. A total of 134 (100 MRgFUS; 34, sham) subjects were included in an intent to treat analysis excluding screen failures. Blinding of sham subjects was excellent with 96 and $88 \%$ on the MRgFUS and sham arms respectively indicating they believed they had received actual treatment.

Baseline patient demographic characteristics were similar in the two arms with the exception of gender with more females randomized to sham (81\%) than MRgFUS (56\%). However, this imbalance did not meaningfully affect the efficacy results. Disease characteristics were similar between groups.

All study endpoints were met. Three months after treatment $67 \%$ of MRgFUS subjects experienced pain palliation compared to $21 \%$ of sham subjects $(p<0.001)$. A secondary efficacy endpoint measured the NRS as both change from baseline to month 3 and change from baseline to other study visits to evaluate time course of success. NRS scores in the MRgFUS arm decreased a mean of 3.8 points from baseline versus 0.7 points in the sham arm. Improvement in the MRgFUS arm was noted as early as day 1 with continued improvement through the first month with the improvement in pain maintained through study completion.

Quality of life was also markedly impacted by MRgFUS in this patient population. Overall BPI-QOL score mean change from baseline through month 3 was 2.4 points for the MRgFUS arm and 0.1 points for the Sham arm $(p<0.001)$. Improvement in quality of life was immediate with significant mean improvement in BPI-QOL for MRgFUS subjects of 1.1 on day 1 post-treatment with continued improvement through month 2 .

Seventeen subjects from the sham arm elected to crossover and receive MRgFUS treatment after un-blinding due to sham treatment failure. Seventy-six percent responded to MRgFUS treatment with mean improvement of 4.9 points on the NRS and 2.4 points improvement on the BPI-QOL. The safety profile of MRgFUS was excellent. Transient sonication pain during treatment was the most common adverse event.
The phase III trial results support and expand upon the findings of the phase I-II studies. Noting all subjects in this phase III trial either had an inadequate response to radiation or were unsuitable candidates for radiation, a clinically significant response rate of $67 \%$ was achieved. Nearly one quarter of MRgFUS subjects experienced complete pain response. Importantly, response to MRgFUS was typically rapid with most responders experiencing clinically significant pain relief and improved quality of life within several days of the procedure. Similar to the phase I-II experience, MRgFUS was well tolerated with minimal toxicity. The strong statistical and most importantly clinically significant benefits of MRgFUS for treatment of bone metastases strongly support use of this modality for treatment of localized pain for patients who have persistent or recurrent pain after radiation or for whom radiation therapy is contraindicated.

\section{Future Directions}

Future areas of research include expansion of indications and sites suitable for MRgFUS treatment, applicability of MRgFUS as a primary treatment for painful bone metastases either as stand alone therapy or in combination with radiation, and development of conformal treatment devices. Given the wider availability of USgFUS, research into application of some of the current advantages of MR guidance, treatment monitoring, and verification to ultrasound guided therapy is also warranted. Current clinical trials of MRgFUS are listed in Table 2.

Trials to date have focused on use of MRgFUS as a second-line therapy. The recently completed phase III trial had stringent eligibility criteria that, while necessary to minimize risk of confounding variables impacting study results, are not limitations to use as routine therapy. For instance in order to differentiate response, patients on the phase III trial could have no more than 5 painful lesions of which only one could be treated. Furthermore, the pain associated with the treated lesion had to be at least 2 NRS points greater than other lesions. A strict pain management optimization protocol also had to be followed prior to patients being allowed to move forward with treatment. These restrictive criteria meant to facilitate assessment of the value of MRgFUS in an investigative setting are, however, not limiting to routine clinical practice. As clinical experience with treatment of bone metastases grows it may also be possible to expand use to a greater number of anatomic sites. Use of conformal applicators, now in clinical trials, allows for greater latitude in positioning of the applicator to the treatment site as opposed to a table mounted applicator. This versatility should allow for expanded use of FUS in this patient population. 
Table 2 Current clinical trials of MRgFUS for the treatment of bone tumors

\begin{tabular}{lllllll}
\hline Trial/identifier & Center(s) & Phase & $n$ & Arm(s) & $\begin{array}{l}\text { Tumor: } 1^{\circ} \\
\text { vs. } 2^{\circ}\end{array}$ & $\begin{array}{l}\text { Primary outcome } \\
\text { measures }\end{array}$ \\
\hline NCT00656305 & USA; Israel; Italy; Russia & III & 147 & $\begin{array}{l}\text { MRgFUS } \\
\text { Placebo }\end{array}$ & Pain \\
NCT01640847 & USA & II & 52 & $\begin{array}{l}\text { MRgFUS + Lyso-thermo } \\
\text { sensitive Liposomal }\end{array}$ & 2 & Pain \\
NCT01085565 & Israel & & & Doxorubicin & 2 & Pain \\
NCT01693770 & Rome, Italy & II & 50 & MRgFUS & 2 & Adverse events \\
NCT01309048 & Toronto, Canada & I/II & 18 & MRgFUS & 2 & Pain; adverse events \\
NCT01117246 & Bordeaux, France & I/II & 10 & MRgFUS & 2 & Pain; adverse events \\
NCT00981578 & USA: Stanford; Methodist; UVA & I & 50 & MRgFUS & Adverse events \\
NCT01586273 & India; Netherlands; UK & N/A & 64 & MRgFUS & 2 & Pain \\
\hline
\end{tabular}

MRgFUS magnetic resonance guided focused ultrasound, $N R$ not reported, N/A not applicable

The role of FUS as primary treatment for painful bone metastases also warrants investigation. Additional consideration to the goals of treatment and methods to achieve them is required. Particularly in weight bearing bones, the issue of bone integrity and its relation to tumor regrowth must be accounted for which will likely require more aggressive treatment than required for pain relief alone.

Use of FUS in a multimodality treatment setting is a very intriguing avenue for future research. The biologic benefits to combining nonablative thermal therapy, often referred to as hyperthermia, to radiation is well established [22-27] and there is growing evidence to support use of heat with chemotherapy [27, 28] and immunotherapy [29-33]. Given there is a heated but nonablated rim of tissue with thermal ablation, opportunity to take advantage of thermal biology exist both with ablative and nonablative application of FUS.

\section{Conclusions}

Focused ultrasound, particularly with MR guidance, has been shown to have a clear role in the management of painful bone metastases. A recently completed phase III placebo controlled trial confirmed that excellent rates of durable pain relief and improvement in quality of life for patients with oncologic related bone pain can be achieved with MRgFUS even in the second line treatment setting for whom radiation therapy is ineffective or contraindicated. Ongoing and future studies promise to define an expanded role of focused ultrasound in this patient population in acute need of effective new treatment options.

Disclosure No potential conflicts of interest relevant to this article were reported.

\section{References}

Papers of particular interest, published recently, have been highlighted as:

- Of importance

- Of major importance

1. Buckwalter JA, Brandser EA. Metastatic disease of the skeleton. Am Fam Physician. 1997;55:1761-8.

2. Sabino MA, Mantyh PW. Pathophysiology of bone cancer pain. J Support Oncol. 2005;3:15-24.

3. Nielsen OS, Bentzen SM, Sandberg E, et al. Randomized trial of single dose versus fractionated palliative radiotherapy of bone metastases. Radiother Oncol. 1998;47:233-40.

4. Nielsen OS, Munro AJ, Tannock IF. Bone metastases: pathophysiology and management policy. J Clin Oncol. 1991;9:509-24.

5. Goblirsch MJ, Zwolak PP, Clohisy DR. Biology of bone cancer pain. Clin Cancer Res. 2006;12:6231s-5s.

6. Meuser T, Pietruck C, Radbruch L, et al. Symptoms during cancer pain treatment following WHO-guidelines: a longitudinal follow-up study of symptom prevalence, severity and etiology. Pain. 2001;93:247-57.

7. Ibrahim T, Flamini E, Mercatali L, et al. Pathogenesis of osteoblastic bone metastases from prostate cancer. Cancer. 2010;116: 1406-18.

8. Chow E, Harris K, Fan G, et al. Palliative radiotherapy trials for bone metastases: a systematic review. J Clin Oncol. 2007;25:1423-36.

9. Cleeland CS, Ryan KM. Pain assessment: global use of the Brief Pain Inventory. Ann Acad Med Singapore. 1994;23:129-38.

10. Chow E, Hartsell WF, Roos R, et al. Single-fraction compared with multiple-fraction therapy in treating patients with previously irradiated painful bone metastases. 2012. http://clinicaltrials.gov/ ct2/show/NCT00080912. Accessed 29 Apr 2013.

11. Hartsell WF, Scott CB, Bruner DW, et al. Randomized trial of short- versus long-course radiotherapy for palliation of painful bone metastases. J Natl Cancer Inst. 2005;97:798-804.

12. Serlin RC, Mendoza TR, Nakamura Y, et al. When is cancer pain mild, moderate or severe? Grading pain severity by its interference with function. Pain. 1995;61:277-84.

13. Torrance GW, Feeny D, Furlong W. Visual analog scales: do they have a role in the measurement of preferences for health states? Med Decis Making. 2001;21:329-34. 
14. Clarke B. Normal bone anatomy and physiology. Clin J Am Soc Nephrol. 2008;3(Suppl 3):S131-9.

15. Chen $\mathrm{W}$, Zhu H, Zhang L, et al. Primary bone malignancy: effective treatment with high-intensity focused ultrasound ablation. Radiology. 2010;255:967-78.

16. Li C, Wu P, Zhang L, et al. Osteosarcoma: limb salvaging treatment by ultrasonographically guided high-intensity focused ultrasound. Cancer Biol Ther. 2009;8:1102-8.

17. Li C, Zhang W, Fan W. Noninvasive treatment of malignant bone tumors using high-intensity focused ultrasound. Cancer. 2010; 116:3934-42.

18. - Gianfelice D, Gupta C, Kucharczyk W, et al. Palliative treatment of painful bone metastases with MR imaging-guided focused ultrasound. Radiology. 2008; 249:355-63. Early-phase study where MRgFUS was used for the treatment of bone metastases.

19. - Catane R, Beck A, Inbar Y, et al. MR-guided focused ultrasound surgery (MRgFUS) for the palliation of pain in patients with bone metastases-preliminary clinical experience. Ann Oncol. 2007; 18:163-167. Early-phase study where MRgFUS was used for the treatment of bone metastases.

20. - Liberman B, Gianfelice D, Inbar Y, et al. Pain palliation in patients with bone metastases using MR-guided focused ultrasound surgery: a multicenter study. Ann Surg Oncol. 2009; 16:140-46. Early-phase study where MRgFUS was used for the treatment of bone metastases.

21. • InSightec. ExAblate (Magnetic Resonance-guided Focused Ultrasound Surgery) Treatment of Metastatic Bone Tumors for the Palliation of Pain, NCT00656305. [Completed clinical trial]. 2012. http://clinicaltrials.gov/show/NCT00656305. Accessed 26 Nov 2012. Currently, this is the only Phase III evidence available for MRgFUS for the treatment of bone metastases. Notably, it is one of the few studies with detailed pain assessment.

22. Dewey WC, Thrall DE, Gillette EL. Hyperthermia and radiation-a selective thermal effect on chronically hypoxic tumor cells in vivo. Int J Radiat Oncol Biol Phys. 1977;2:99-103.
23. Westra A, Dewey WC. Variation in sensitivity to heat shock during the cell-cycle of Chinese hamster cells in vitro. Int J Radiat Biol Relat Stud Phys Chem Med. 1971;19:467-77.

24. Gerweck LE, Nygaard TG, Burlett M. Response of cells to hyperthermia under acute and chronic hypoxic conditions. Cancer Res. 1979;39:966-72.

25. Hahn GM, Shiu EC. Adaptation to low pH modifies thermal and thermo-chemical responses of mammalian cells. Int J Hyperthermia. 1986;2:379-87.

26. Henle KJ, Leeper DB. Combinations of hyperthermia (40 degrees, 45 degrees C) with radiation. Radiology. 1976;121:451-4.

27. Kano E. Hyperthermia and drugs. In: Overgaard J, editor. Hyperthermia oncology. London: Taylor \& Francis; 1985. p. $277-82$.

28. Issels RD, Lindner LH, Verweij J, et al. Neo-adjuvant chemotherapy alone or with regional hyperthermia for localised highrisk soft-tissue sarcoma: a randomised phase 3 multicentre study. Lancet Oncol. 2010;11:561-70.

29. Asea A, Kraeft SK, Kurt-Jones EA, et al. HSP70 stimulates cytokine production through a CD14-dependant pathway, demonstrating its dual role as a chaperone and cytokine. Nat Med. 2000;6:435-42.

30. Srivastava PK, Maki RG. Stress-induced proteins in immune response to cancer. Curr Top Microbiol Immunol. 1991;167: 109-23.

31. Udono H, Srivastava PK. Heat shock protein 70 -associated peptides elicit specific cancer immunity. J Exp Med. 1993;178: 1391-6.

32. Udono H, Srivastava PK. Comparison of tumor-specific immunogenicities of stress-induced proteins gp96, hsp90, and hsp70. J Immunol. 1994;152:5398-403.

33. Udono H, Levey DL, Srivastava PK. Cellular requirements for tumor-specific immunity elicited by heat shock proteins: tumor rejection antigen gp96 primes CD8 + T cells in vivo. Proc Natl Acad Sci USA. 1994;91:3077-81. 